\title{
In vivo measurements of blood viscosity and wall stiffness in the carotid using PC-MRI
}

\author{
Stephane Avril* - Jonathan M. Huntley ${ }^{* *}$ - Rhodri Cusack*** \\ * Centre Ingénierie et Santé - PECM CNRS UMR 5146 \\ Ecole Nationale Supérieure des Mines \\ 158 cours Fauriel \\ F-42023 Saint-Etienne cedex 2, France \\ ** Wolfson School of Mechanical and Manufacturing Engineering \\ Loughborough University \\ Loughborough, LE11 3TU, United Kingdom \\ *** MRC Cognition and Brain Sciences Unit \\ 15 Chaucer Road \\ Cambridge, CB2 2EF, United Kingdom
}

\begin{abstract}
A method is proposed for deducing blood viscosity and wall stiffness in the carotid from Phase-Contrast MRI data. The approach is based on Womersley's model of blood flow derived from the resolution of the Navier-Stokes equations, assuming blood as a Newtonian fluid and the artery as a linear elastic cylindrical pipe. After presenting its principle, the approach is applied to the experimental data obtained on a single volunteer. Promising results are obtained.

RÉSUMÉ. Une méthode est proposée pour identifier la viscosité du sang et la rigidité de paroi dans l'artère carotide à partir de mesures réalisées par IRM en contraste de phase. La méthode repose sur le modèle d'écoulement de Womersley déduit de la solution des équations de Navier-Stokes pour un fluide newtonien s'écoulant dans une conduite cylindrique élastique. Après présentation du principe, la méthode est appliquée sur des données expérimentales obtenues sur un seul patient. Des résultats satisfaisants sont obtenus.

KEYWORDS: MRI, identification, blood viscosity, artery elasticity, Womersley flow.

MOTS-CLÉS: IRM, identification, viscosité du sang, élasticité des artères, écoulement de Womersley.
\end{abstract}

DOI:10.3166/EJCM.18.9-20 @ 2009 Lavoisier, Paris

EJCM - 18/2009. Numerical models in biomechanics, pages 9 to 20 


\section{Introduction}

Cardiovascular disease remains the leading cause of mortality in industrialized nations. An increase in arterial wall stiffness appears to be a common pathological pathway for the many factors that lead to initiation and progression of the vascular changes associated with cardiovascular disease. An accurate measurement of this stiffness is therefore of real interest. Such a measurement is however difficult to separate from the measurement of blood viscosity as both parameters are the main ones affecting the form of blood flow in the arteries.

Numerical models are increasingly commonplace in the field of biomechanics. The simulation of blood flow (Maurits et al., 2007) or of artery deformations (Li et $a l ., 2006)$ is essential for understanding phenomena such as plaque formation and failure. For that, accurate estimates of the mechanical properties governing the artery deformations and blood flow are required. The aim of this paper is to present a novel method that can provide these properties in a non intrusive way using Magnetic Resonance Imaging (MRI).

Classically, different ways exist for characterizing arterial wall stiffness (Hansen et al., 1995; Fronek et al., 1976; Asmar et al., 1995; Stephanis et al., 2003; Liepsch, 2002). The most common way is to use the pulse wave velocity (PWV), denoted $c_{r}$, which satisfies, within the framework of a few assumptions, the MoensKorteweg equation: $c_{r}^{2}=E h / 2 \rho R_{0}$, where $E$ is the Young's modulus of the arterial wall; $h$ is the wall thickness; $R_{0}$ is the arterial radius (at rest); $\rho$ is the blood density (McDonald, 1974). The PWV is calculated from measurements of pulse transit time and the distance travelled by the pulse between two recording sites, using manometers. When pressure measurements are not possible, ultrasound or MRI can be used for measuring the diameter of the artery and the velocity of the blood. These measurements have sometimes been coupled to pressure measurements in the literature for deriving the PWV. For example, with ultrasound, the measurement of the diameter or the velocity (Doppler) can improve the identification of the PWV, and help to assess both $R_{0}$ and $h$ (Maurits et al., 2007; Selzer et al., 2001; Hardt et al., 1999). But no experimental studies have been found where the PWV and consequently the artery elastic modulus could be identified without using pressure measurements.

We show in this study that it is possible to measure these quantities with only shape and velocity measurements, and show a practical application. A more sophisticated model, still compatible with the Moens-Korteweg equation, is used. Moreover, a technique to identify the blood viscosity along with the elastic modulus of the artery will be proposed. The non-intrusive identification of blood viscosity is original. Indeed, blood viscosity is a very important parameter, usually measured using viscometers (Shin et al., 2002). This means that blood needs to be taken from the human body before being tested, requiring the use of anticoagulants for avoiding blood coagulation. The effects of the anticoagulants on blood viscosity are almost unknown (Shin et al., 2002). Accordingly, there is a potential interest in characterizing blood viscosity in its real environment thanks to medical imaging. 
The main measurement techniques that are available in clinics are MRI and ultrasound. MRI can provide a much higher resolution than ultrasound imaging (Draney et al., 2002; Li et al., 2006; Moreno et al., 1998). Comparing the velocity profiles (Maurits et al., 2007) with the ones shown further in this paper demonstrates that the accuracy of velocity measurements using ultrasound is typically worse than that which can be obtained with Phase-Contrast Magnetic Resonance Imaging (PC-MRI). Moreover, the local spatial resolution of ultrasound imaging techniques is still not high enough to reach a sufficient accuracy for the wall elasticity (Maurits et al., 2007). Ultrasound transducers are much more widespread than MRI scanners so this may explain why studies using MRI are so scarce. However, the recent proliferation of MRI scanners in clinics, hospitals and research centres may induce an increase of the use of MRI in the domain of cardiovascular medicine. Moreover, the measurement time is not really an issue with modern MRI scanners as the whole velocity data required in our approach can be obtained in less than five minutes. For all these reasons, PC-MRI was chosen as the measurement technique to provide the data required for an accurate identification of blood viscosity and wall stiffness in the carotid.

\section{Basic equations of blood flow in the carotid}

\subsection{Definitions and equations of the artery wall motions}

The model used here is based on the assumption of homogenous, Newtonian, incompressible (with constant density $\rho$ ) fluid (Lighthill, 2002; Lagrée, 2000). The viscosity $\mu$ is also assumed constant. These assumptions may be appropriate for blood if the arteries are wide enough (Lagrée, 2000) and if we neglect density fluctuations of the blood (incompressibility is very often considered as a relevant assumption for modelling blood flow).

The flow is assumed to be axi-symmetrical and gravity effects are neglected. The flow is described by the longitudinal $U(r, x, t)$ and the radial $V(r, x, t)$ components of the velocity vector $\mathbf{u}$. Coordinates $x$ and $r$ denote respectively the longitudinal and axial coordinates, whereas $t$ denotes time.

The pressure in the fluid is assumed constant across any slice of the artery, only depending on $x$ and $t$. It is denoted $p(x, t)$. The local radius of the artery is denoted $R(x, t)$. Both are linked by the elasticity of the artery wall. The artery wall behaves, to a first approximation, like an elastic membrane in plane stress with a Young's modulus denoted $E$. The acceleration forces of the artery wall are neglected compared to the tension forces. Using standard force balance arguments for pressurized elastic cylindrical pipes (Lagrée, 2000), one ends up with the the following equation linking $R$ and $p$ :

$$
\frac{1}{R} \frac{\partial R}{\partial p}=\frac{R_{0}}{h E}
$$




\subsection{General form of blood flow}

Gradients are assumed to remain small so that the equations can be simplified by linearization. The following Navier-Stokes equations are obtained in cylindrical coordinates:

the linearized momentum equation: the local continuity equation:

$$
\rho \frac{\partial U}{\partial t}=-\frac{\partial p}{\partial x}+\mu\left[\frac{\partial^{2} U}{\partial r^{2}}+\frac{1}{r} \frac{\partial U}{\partial r}\right] \quad \frac{\partial U}{\partial x}=-\frac{1}{r} \frac{\partial(r V)}{\partial r}
$$

where $\mu$ is the blood viscosity.

The problem to solve is a problem with moving boundaries, as $R$ changes with the pressure variations according to Equation [1]. For solving this problem coupling artery deformation and the blood flow, one has to introduce equations figuring fluidstructure interactions. On the assumption that there is no slippage at the artery wall, the boundary conditions at $r=R$ are $U=0$ and $V=\partial R / \partial t$ for all $x$ and $t$. This means that there is no slippage at the artery wall.

Due to the heart beats, $U, R$ and $p$ are forced to vary periodically with $t$ and $x$ (forced regime). $U$ is a periodic function of $t$ and $x$, and one may therefore write it as a Fourier series. Using this Fourier decomposition, Womersley (Lagrée, 2000) found the following solution:

$U(x, r, t)=U_{0}\left[1-(r / R)^{2}\right]+2 \mathcal{R}\left\{\sum_{n=1}^{+\infty} \frac{p_{n}}{\rho c} \frac{1-\frac{J_{0}\left(i^{3 / 2} \alpha_{n} r / R\right)}{J_{0}\left(i^{3 / 2} \alpha_{n}\right)}}{1-\frac{1}{J_{0}\left(i^{3 / 2} \alpha_{n}\right)}} \exp \left[\frac{2 i n \pi}{T}(t-x / c)\right]\right\}$

where $\mathcal{R}$ means "the real part" of a complex number, $i$ is the square root of $-1, T$ is the pulse period, $J_{0}$ is Bessel's function of first type and $\alpha_{n}$ is the Womersley number corresponding to order $n: \alpha_{n}=R_{0} \sqrt{2 \pi n \rho /(\mu T)} ; c$ is the complex celerity: its real part is the PWV denoted $c_{r}$ and its imaginary part, denoted $c_{i}$, accounts for the decay of the travelling wave due to viscous effects (Lagrée, 2000).

The $p_{n}$ 's are, up to a multiplicative constant, the Fourier coefficients (complex numbers) of the pressure variations that may be written:

$$
p(x, r, t)=p_{0}+g_{0} x+2 \mathcal{R}\left\{\sum_{n=1}^{n=+\infty} \frac{p_{n}}{1-\frac{1}{J_{0}\left(i^{3 / 2} \alpha_{n}\right)}} \exp \left[\frac{2 i n \pi}{T}(t-x / c)\right]\right\}
$$

where $g_{0}$ is a constant pressure gradient. The first term in Equation [3] is constant in time (the dc term). It is the steady solution found by Poiseuille (Lagrée, 2000) for a 
Newtonian fluid flowing with the constant pressure gradient $g_{0}$ in Equation [4]. The factor of 2 before the fundamental and harmonics in Equations [3] and [4] accounts for the fact that Fourier coefficients corresponding to negative $n$ are omitted (even function). The $U_{0}$ coefficient in Equation [3] and the $g_{0}$ coefficient in Equation [4] are linked by the following equation: $U_{0}=g_{0} R^{2} /(4 \mu)$. Therefore, if $g_{0}$ was known, it would be very easy to deduce the blood viscosity $\mu$. However, we cannot measure the pressure gradient $g_{0}$ in the carotid using standard manometers, so another way of identifying $\mu$ will be proposed.

\subsection{Identification of blood viscosity}

Let us assume that a measurement of the velocity profiles through a given slice at abscissa $x_{0}$ is available throughout a pulse period. MRI measurements can provide these profiles, as will be shown later. Let $U\left(x=x_{0}, r, t\right)$ denote the measured velocities at any $r$ and $t$. A finite number of relative radii $\eta=r / R$, evenly distributed between 0 and 1 , is defined. At each $\eta, U$ is Fourier transformed along the time axis, providing the Fourier coefficients $\hat{U}_{n}(\eta)$. Then, for a given value of blood viscosity, the deviation between the derived $\hat{U}_{n}(\eta)$ and the Fourier coefficients of Equation [3] is minimized in the least-squares sense, for any $\eta$ and any $n>0$ (limited to $n \leq 8$ in practice), yielding the $p_{n}$ 's. These $p_{n}$ values only depend on the arbitrary choice of $\mu$. Subsequently, model velocity profiles that have the form of Equation [3] are reconstructed with the deduced $p_{n}$. Accordingly, the remaining deviation between the modelled velocity profiles and the measured ones only depends on $\mu$. This deviation is then minimized in the least-squares sense, with $\mu$ being the controlling variable. The minimization of this cost function is achieved using the Nelder Mead algorithm, providing blood viscosity $\mu$ in no more than 20 iterations in practice.

\subsection{Identification of the PWV and the arterial wall stiffness}

By integrating the equation of continuity (Equation [2]) over a whole slice of the artery, and using the equality $\partial / \partial t=-c \partial / \partial x$ (valid in the forced regime, see (Lagrée, 2000)), one obtains:

$$
\int_{0}^{R} \int_{0}^{2 \pi} U\left(x_{0}, r, t\right) r \mathrm{~d} r \mathrm{~d} \theta=J\left(x_{0}, t\right)=c S\left(x_{0}, t\right)+K
$$

where $J\left(x_{0}, t\right)$ is the arterial flow and $S\left(x_{0}, t\right)$ is the area of the arterial cross section. Equation [5] is a classical equation of fluid dynamics in elastic pipes (Lighthill, 2002). Then, by minimization in the least squares sense, it is possible to identify $c$ from the data if both $J$ and $S$ are measured at different times between two heart beats. One has 
to bear in mind that $c$ should be a complex number (Lagrée, 2000). The resolution is achieved in the real space by minimizing over a period the following cost function:

$$
\mathcal{F}(\gamma, \tau, K)=\frac{1}{T} \int_{0}^{T}\left[J\left(x_{0}, t\right)-\gamma S\left(x_{0}, t-\tau\right)+K\right]^{2} \mathrm{~d} t
$$

where $\gamma$ is the scaling coefficient between $J$ and $S$ (magnitude of complex $c$ ), $\tau$ is the time delay between $J$ and $S(2 \pi \tau / T$ is the angle of complex $c)$ and $K$ is a constant. In practice, $U$ and $R$ are obtained from the MRI data. Then, the values of $J$ and $S$ are deduced from $U$ and $R$. After the minimimization of $\mathcal{F}(\gamma, \tau, K)$, the PWV can be deduced straight forwardly as being $c_{r}=\gamma \cos (2 \pi \tau / T)$.

Knowing the PWV, the elastic modulus of the arterial wall can be deduced using the Moens-Korteweg equation (McDonald, 1974).

\section{Artery wall tracking and blood velocity measurements using MRI data}

\subsection{Principle of Flow Sensitive Phase-Contrast MRI}

Phase contrast MR angiography was used to quantify flow velocity (Hornak, 2007). In this sequence, the flow is encoded in the phase rather than the magnitude of the MRI signal. Between Radio-Frequency (RF) excitation and imaging, velocity encoding is performed by applying two equal and opposite gradients with a short delay between them. If the material within a voxel is stationary, then the phase shift caused by one gradient will be cancelled by the equal and opposite phase shift due to the other, and the net phase change measured will be zero. However, if material moves along the direction of the gradient, then it will gain a net phase shift proportional to this component of velocity.

Blood and the vessel wall also have distinct $T_{1}$ and $T_{2}$ relaxation parameters, giving clear contrast in the magnitude images in the sequence (MacRobbie et al., 2003). We used this to estimate the location of the artery contour, and to generate a mask to remove all voxels outside it. The mask was applied to the phase maps for investigating the velocity profiles and identifying the elastic and viscous parameters. Although it is also possible to generate a mask from the phase images, we found that in the magnitude images the gradient at the vessel wall was higher and the signal outside the artery less noisy, enhancing contour estimation.

\subsection{Geometry and velocity measurements around a volunteer's carotid}

The scanner used in our study was a 3T Siemens Tim Trio system (MRC Cognition and Brain Sciences Unit, Cambridge, UK). A 2D spin-echo FLASH sequence was used to acquire a single $3 \mathrm{~mm}$ thick slice of a lying volunteer's neck with a matrix size 
of $240 \times 256$ giving in-plane dimensions $0.39 \times 0.39 \mathrm{~mm}^{2}$. A cine sequence, with one segment per cycle was used to acquire the temporal evolution of the flow throughout the pulse. Heart beats were detected by the measurement of blood flow in a patient's finger with near infrared spectrometry. The cine data were reconstructed to give 50 snapshots evenly distributed throughout the cardiac cycle, corresponding to a mean sampling frequency of $61.5 \mathrm{~s}^{-1}(T=0.81 \mathrm{~s})$. The repetition time was $\mathrm{TR}=64 \mathrm{~ms}$ and the echo time was TE=5.4 ms. The velocity encoding gradient was $70 \mathrm{~cm} / \mathrm{s}$ per $\pi \mathrm{rad}$ in the direction perpendicular to the plane (e.g., head-foot). The phase of the signal was digitized with a 12 bits resolution (integer numbers between 0 and 4095) and the magnitude 16 bits (0-65535). The magnitude images were used to characterise geometry and the phase images the velocity at each moment in time.

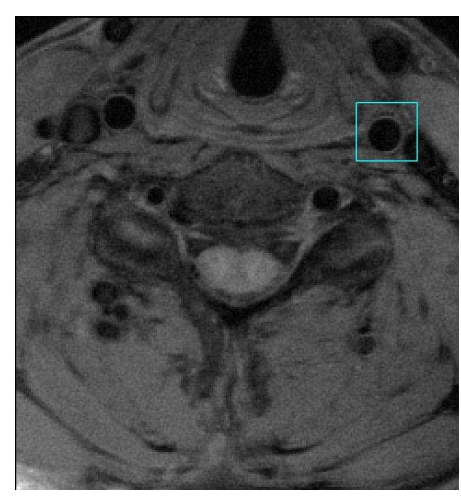

(a) Whole neck cross-section (blackblood sequence)

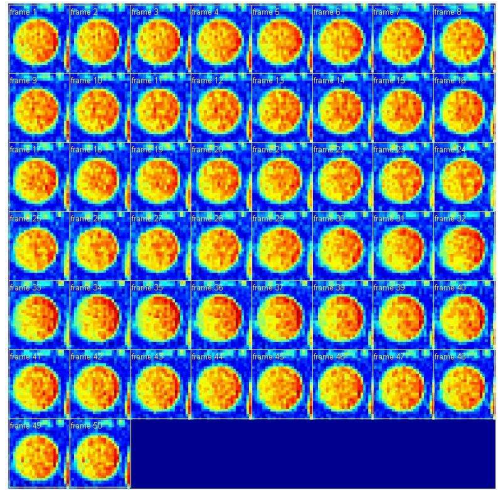

(b) Frames of signal magnitude

Figure 1. Images of the MRI signal magnitude

\subsection{Definition of the artery mask}

The magnitude of the signal is well suited for determining the edges of the artery because, due to the large quantity of blood flowing in the artery, the magnitude of the signal is larger inside the artery than outside (Figure 1b).

Thanks to this profile, the contour of the artery is detected for each frame using the following method in which we assume a cylindrical shape for the artery, for consistency with the assumed flow model. The assumed image model is a "top hat": a constant signal of magnitude $M_{1}$ inside the circular contour and constant signal of magnitude $M_{2}$ outside the contour. We neglect blurring due to finite point spread function of the imaging system in the model. The problem is to choose the centre, the radius, and the values of $M_{1}$ and $M_{2}$ so as to minimize the least-squares deviation between the data and the "top hat" model. Minimisation is achieved using the Gauss-Newton method. 
The masks deduced for all frames were applied to the signal phase maps, which have been also unwrapped and scaled to velocity for all the frames. The deduced velocity maps are shown in Figure 2a.

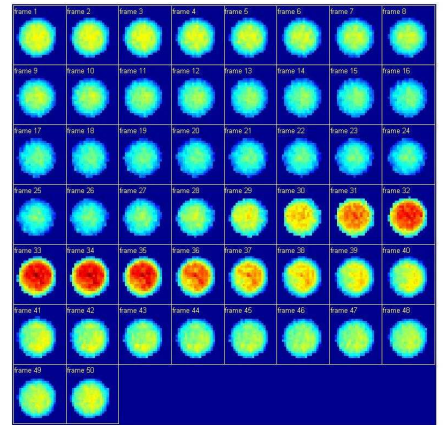

(a) 50 velocity frames in the carotid

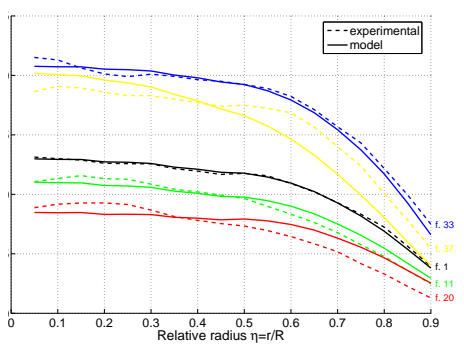

(b) Profiles of the velocity for a few frames.

Figure 2. Measured velocity profiles during a heart beat and comparison of the profiles with the identified model for a few frames

\section{Results and discussion}

\subsection{PWV identification}

Plotting $J$ against $S$ for all the frames, one obtains a loop (Figure 3a). Because of the viscous behaviour of blood, $J$ and $S$ are not in phase, which explains the loop shape. Accordingly, $c$ is a complex number; its real part is the PWV and its imaginary part accounts for the decay of the travelling wave due to viscous effects (Lagrée, 2000). The minimimization of $\mathcal{F}$ defined in Equation [6] leads to the value $c_{r}=2.73 \mathrm{~m} / \mathrm{s}$.

There remain deviations between the experimental values of $J$ and the supposedly modelled values $J=c S+K$ (Figure 3). This may mean that the phase lag between flow and area is frequency dependent. We would need to analyze the Fourier components of $J$ and $S$ to investigate this point further, but this is beyond the scope of this paper.

The value $c_{r}=2.73 \mathrm{~m} / \mathrm{s}$ is fairly consistent with values that are reported in the literature. The PWV ranges usually between 3 and $10 \mathrm{~m} / \mathrm{s}$ for the femoral artery or the aorta (Lagrée, 2000), and thus it is expected to be slightly lower for the carotid due to its smaller size. The consistency of the result is promising regarding the relevancy of the assumptions made for deriving the model, especially the assumption of the forced regime. Tests on other patients are envisaged in the near future to check on the repeatability of the identified value of $c_{r}$.

Variations of $J$ through a heart beat have been plotted in Figure $3 \mathrm{~b}$. Two peaks are apparent: a large one at $t=530 \mathrm{~ms}$, and a smaller one at $t=800 \mathrm{~ms}$. The large 
one corresponds to the pulse wave induced by the opening of the sigmoid valve in the heart, with the smaller one corresponding to the pulse wave induced by the closing of the sigmoid valve. This shape of blood flow is common and in agreement with other published data (Liepsch, 2002).

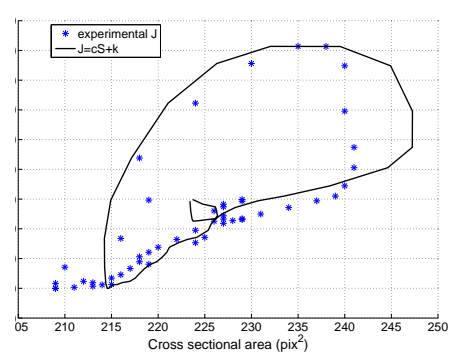

(a) $J$ versus $S$

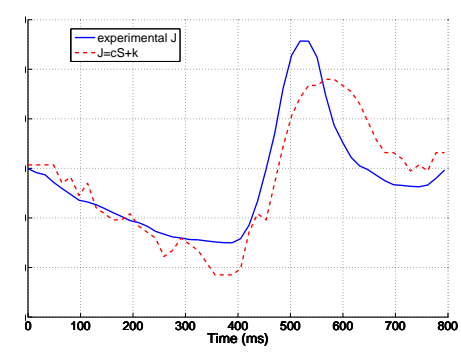

(b) $J$ versus $t$

Figure 3. Variations of global blood flow in the carotid

\subsection{Reconstruction of modelled velocity profiles}

The modelled profiles that best fit the data in the least squares sense have been derived. The corresponding viscosity parameter is $\mu=0.0073 \mathrm{~Pa}$.s. It has been checked that the value of $\mu=0.0073 \mathrm{~Pa}$.s constitutes the only minimum of the cost function. It corresponds to a Womersley number for the fundamental mode ( $n=1$ in Equation [3]) of $\alpha_{1}=3.1$. The modelled flow reconstructed with $\mu=0.0073$ Pa.s has been plotted in Figure $4 \mathrm{a}$ (maps for the 50 frames).

The residuals between the measured velocities and the modelled ones are shown in Figure $4 \mathrm{~b}$. They prove that the model and the data are in reasonable agreement, except between frames 31 and 40 . For those particular frames, corresponding to the time when the pulse wave induced by the opening of the sigmoid valve is passing, it seems that there is an asymmetry in the velocity profile. This asymmetry is not compatible with the Womersley model, so it could not be reproduced analytically. The asymmetry may be induced by local curves of the artery axis, or by a bifurcation, that are not taken into account in the model (the model assumes that the artery axis is a straight line).

Asymmetry of the flow after a bifurcation has already been observed using numerical models (Liepsch, 2002). Computational Fluid Dynamics (CFD) would be necessary to take the bifurcation into account in the model. This would mean that the resolution of the inverse problem would be numerical and not analytical any longer. This prospect may have to be investigated in the future, by measuring the velocity in different slices and reconstructing a 3D model of the carotid and the blood flowing in it. 


\subsection{Blood viscosity and artery elasticity identification}

The obtained result $\mu=0.0073$ Pa.s is consistent with values found in the literature regarding blood viscosity: values of $\mu$ varying between 0.003 and 0.03 Pa.s have been reported (Shin et al., 2002). However, it is reported in the literature that $\mu$ can significantly vary with the shear strain rate, and also with blood composition. Unfortunately, these variations, involving a non Newtonian behaviour of blood, cannot be characterized with our approach. Our approach only provides an average value of blood viscosity in the conditions within which the measurements are achieved.

Nevertheless, the measured profiles and the identified blood viscosity can be employed for quantifying the shear stresses acting on the artery walls. The values of the shear stresses are of great interest for clinical purposes as they may directly affect the possible development of plaques in the artery (Li et al., 2006).

From $c$ and the artery wall thickness $h=0.5 \mathrm{~mm}$ (measured from the "black blood" image in Figure 1a), the artery wall elastic modulus was estimated using the MoensKorteweg equation and gave the value $E=99 \mathrm{kPa}$. This is somewhat lower than the values from other studies. Using a B-scan method, (Stephanis et al., 2003) found a Young modulus of about $300 \mathrm{kPa}$. (Selzer et al., 2001) found moduli of about $600 \mathrm{kPa}$ on various patients. (Selzer et al., 2001) showed that the obtained modulus values could have a standard deviation of about $250 \mathrm{kPa}$.

However, there is a large uncertainty on the parameter $h$ (wall thickness), as this parameter was not measured precisely during the MRI sequences. It is more relevant to compare the coefficient of elasticity $k=E h / R_{0}$ which took the value of $14.9 \mathrm{kPa}$ for our volunteer. For comparison, (Stephanis et al., 2003) found a coefficient of elasticity of about $15 \mathrm{kPa}$. Therefore, it seems likely that the underestimation of $E$ in our results is mainly induced by errors in the estimation of the wall thickness. An accurate measurement of this quantity will be required for further experiments.

Our results should also be verified by repeating the measurement on the same volunteer, for characterizing the repeatability and the robustness of the method. Moreover, an application of our approach on several volunteers and also on different locations of the carotid would be required for a more complete validation.

\section{Conclusion}

This study has shown that it is possible to identify simultaneously the wall stiffness and the viscosity of blood in the carotid by using velocity profiles provided by PC-MRI. The approach is based on an analytical model of the flow derived from a particular solution of the Navier-Stokes equations, assuming blood as a Newtonian fluid and the artery as a linear elastic cylindrical pipe.

The approach requires a measurement of both the field of blood velocities and the variations of the artery radius during a heart beat. Both measurements were obtained from magnitude and phase maps provided by a spin-echo pulse sequence with a ve- 
locity encoding gradient. Eventually, the identified values of blood viscosity and wall stiffness, obtained on a single volunteer, were in good agreement with values reported in the literature. This is promising for the development of our approach, even if repeatability studies are now required.

The accuracy of the approach can be improved by refining the spatial resolution in further measurements. MRI sequences providing a better spatial resolution can be found in the literature (Draney et al., 2002; Li et al., 2006). Reproducing one of the sequences employed in (Draney et al., 2002) or (Li et al., 2006) would significantly improve the accuracy of our identification approach. The measurement of accurate in-plane velocities could also be targeted. This would provide measurements of the arterial wall deformation throughout a heart beat, and make possible an extension of our approach to arteries that are not round, and even to arteries with plaques.

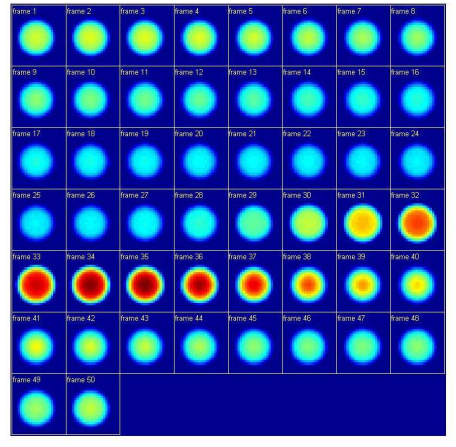

(a) Modelled velocity profiles

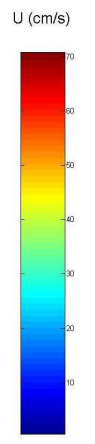

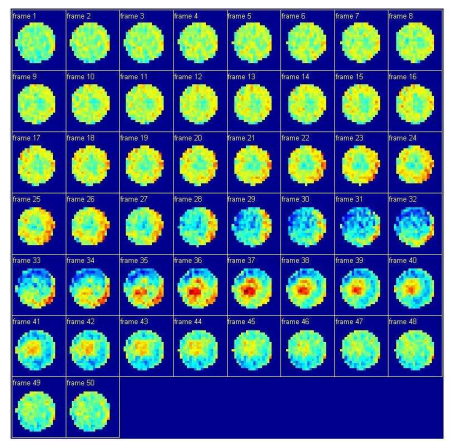

(b) Deviation between data and model

Figure 4. Modelled velocity after identification of the blood viscosity and comparison with the experimental data

Acknowledgements

We are pleased to acknowledge useful technical advice from M. Graves. J.M. Huntley is also grateful to the Royal Society and Wolfson Foundation for a Royal Society-Wolfson Research Merit Award.

\section{References}

Asmar R., Benetos A., Topouchian J., Laurent P., Pannier B., Brisac A., Target R., Levy B., «Assessment of Arterial Distensibility by Automatic Pulse Wave Velocity Measurement », Hypertension, vol. 26, p. 485-490, 1995. 
Draney M., Herfkens R., Hugues T., Pelc N., Wedding K., Zarins C., Taylor C., « Quantification of Vessel Wall Cyclic Strain Using Cine Phase Contrast Magnetic Resonance Imaging », Annals of Biomedical Engineering, vol. 30, p. 1033-1045, 2002.

Fronek K., Schmid-Shoenbein G., Fung Y., « A non-contact method for the three-dimensional analysis of vascular elasticity in vivo and in vitro », Journal of applied physiology, vol. 40, p. 634-637, 1976.

Hansen B., Menkis A., Vesely A., « Longitudinal and radial distensibility of the porcine aortic root», Annals of thoracic surgeons, vol. 60, p. 384-390, 1995.

Hardt S., Just A., Bekeredjian R., Kubler W., Kirchheim H., Kuecherer H., « Aortic pressurediameter relationship assessed by intravascular ultrasound: experimental validation in dogs », IVUS for measurement of aortic wall motion, The American physiological society, H1078-H1085, 1999.

Hornak J., The basics of MRI, http://www.cis.rit.edu/htbooks/mri, 2007.

Lagrée P.-Y., « An inverse technique to deduce the elasticity of a large artery », The European Physical Journal - Applied Physics, vol. 9, p. 153-163, 2000.

Li Z.-Y., Howarth S., Trivedi R., U-King-Im J., Graves M., Brown A., Wang L., Gillard J., « Stress analysis of carotid plaque rupture based on in vivo high resolution MRI », Journal of Biomechanics, vol. 39, p. 2611-2622, 2006.

Liepsch D., « An introduction to biofluid mechanics - Basic models and applications », Journal of Biomechanics, vol. 35, p. 415-435, 2002.

Lighthill J., Waves in fluids, Cambridge Mathematical Library, Cambridge, UK, 2002.

MacRobbie D., Moore E., Graves M., Prince M., MRI: From Picture to Proton, Cambridge University Press, 2003.

Maurits N., G.E. L., A.E.P. V., « The influence of vessel wall elasticity and peripheral resistance on the carotid artery flow wave form: A CFD model compared to in vivo ultrasound measurements », Journal of Biomechanics, vol. 40, p. 427-437, 2007.

McDonald D., Blood Flow in Arteries, Edward Arnold Publishers Inc., London, UK, 1974.

Moreno M., Moore J., Meuli R., « Cross-sectional deformation of the aorta as measured with magnetic resonance imaging », Journal of Biomechanical Engineering, Transactions of the ASME, vol. 120, p. 18-21, 1998.

Selzer R., Mack W., Lee P., Kwong-Fu H., Hodis H., « Improved common carotid elasticity and intima-media thickness measurements from computer analysis of sequential ultrasound frames », Atherosclerosis, vol. 154, p. 185-193, 2001.

Shin S., Keum D.-Y., « Measurement of blood viscosity using mass-detecting sensor », Biosensors and Bioelectronics, vol. 17, p. 383-388, 2002.

Stephanis C., Mourmouras D., Tsagadopoulos D., « On the elastic properties of arteries », Journal of Biomechanics, vol. 36, p. 1727-1731, 2003. 